\title{
Deep Learning Convolutional Neural Network for Defect Identification and Classification in Woven Fabric
}

\section{Subrata Das, Sundaramurthy S, Aiswarya M, Suresh Jayaram}

\begin{abstract}
Inspection is the most important role in textile industry which declares the quality of the apparel product. Many Industries were improving their production or quality using Artificial Intelligence. Inspection of fabric in textile industry takes more time and labours. In order to reduce the number of labours and time taken to complete inspection, computerized image processing is done to identify the defects. It gives the accurate result in less time, thereby saves time and increases the production. The convolutional neural network in deep learning is mainly used for image processing for defect detection and classification. The high quality images are given as input, and then the images were used to train the deep learning neural network. Thewovenfabricdefects such as Holes, Selvedge tails, Stains, Wrong drawing and Snarlswere identified by using Convolutional Neural Network. The sample images were collected from the SkyCotex India Pvt.Ltd. The sample images were processed in CNN based machine learning ingoogle platform; the network has a input layer, $n$ number of hidden layer and output layer. The neural network is trained and tested with the samples and the result obtained is used to calculate the efficiency of defect identification.
\end{abstract}

Key words: Convolutional Neural Network, Image processing, Snarl, Selvedge tails, Stains, Deep learning.

\section{INTRODUCTION}

Identifying the defects in the fabrics is a very important process in the textile manufacturing industries as it affects the quality of the fabrics manufactured by the industries. Usually fabric inspection in textile industries is done by human. In manual inspection systems, the trained labours have a chance of finding very less percentage of number of defects whereas automated defect detection increases this number to greater extent. Human vision inspection is not enough accurate due to fatigue and tediousness. This may cause delay and also reduces the inspection efficiency.The highlytrained inspector can detectonly6075\%ofdefectintextile industries ( Everingham and Van gool 2015).Initially, the defects in the fabrics are found manually and only around $60 \%$ of the defects are identified.

Manuscript received on 26 March 2021 | Revised Manuscript received on 07 April 2021 | Manuscript Accepted on 15 April 2021 | Manuscript published on 30 April 2021.

* Correspondence Author

Subrata Das, Professor, Fashion Technology, B.I.T., Sathyamangalam, TN, India.

Sundaramurthy S*, Associate Professor, Department of Information Technology,Bannari Amman Institute of Technology, Tamilnadu, India.

Aiswarya M,Pursuing,Bannari Amman Institute of Technology Fashion Technology,Tamilnadu, India.

Suresh Jayaram, Directors, Skycotex India pvt ltd, Tamilnadu, India.

(C) The Authors. Published by Lattice Science Publication (LSP). This is an open access article under the CC-BY-NC-ND license (http://creativecommons.org/licenses/by-nc-nd/4.0/)
The quality of the fabric can be increased by reducing the number of defects. Duetothisanautomaticsystem is designed and developed to detect defect on the surface of the manufacturing product at fast rate. Also the defect in textile products reduces its price by40\%- 65\%.A Convolutional neural network (CNN) is a class of deep neural networks, most commonly applied to analyzing visual imagery. Deep learning is suitable for real time problems which is nonlinear in nature and have parallel processing ability. The neural networks can handle classification problems with high accuracy (Goyal 2018). There are four parameters, by which the neural networks performance is judged viz. classification accuracy, performance accuracy, type of model used and training time. An application of Convolutional Neural Network in textile industry is increasingintextileprocesses in case of estimationofyarnqualityparameter, classification of knitted and woven fabricetc. This can also be implied in fabric inspection to classify the defects(Habib and Ahmed 2014).The main objective of this research includes reduction in the cost and improvement in the efficiency, to improve overall reliability and reduce the man power and to find the defects in the fabric and notify the workmen.

The Artificial Neural Networks (ANN) is same as the way biological nervous system works, such as brain processes information. ANN mimics models of biological system, which uses numeric and associative processing. In two aspects, it resemblesthe human brain. It acquired knowledge from its environment through a learning process. An automated vision system was presented to detect and classify surface defects in woven fabric (Kang T.J. et al 2001). Fabric texture refers to the feel of the fabric. It is rough, velvety, smooth, soft, silky, lustrous etc. The different textures of the fabric depend upon the types of weaves used. Textures are given to all types of fabrics, cotton, silk, wool, leather, and also to linen. Textile fabric materials are used to prepare different categories and types of Fabric products in the textile industry. Natural fabric and synthetic fabric are the two different classifications of textile fabric. Synthetic fabrics are fairly new and have evolved with the continuous growth in textile industry (Bay Tuytelaars and Van gool2006 ; Kang T.J. et al 2001). Computer vision based, i.e. automated fabric defect inspection systems are thought by many researchers of different countries to resolve these problems. There are two major challenges to be resolved to attain a successful automated fabric defect inspection system. They are defect detection and defect classification. 


\section{Deep Learning Convolutional Neural Network for Defect Identification and Classification in Woven Fabric}

Different techniques has been used for automated fabric defect classification, then show a surveyof classifiers used in automated fabric defect inspection systems, and finally, comparetheclassifiersbyusingperformance metrics(He Zhang and Sun J 2016).

This work is expected to everyusefulfortheresearchersintheareaofautomatedfabricdef ectinspection to understand and evaluate the many potential options in thisfield.Itisthus important toproducedefect freehighqualitygarmentproducts.Inspectionprocessesdoneon fabricindustries are mostly manual hence time consuming. To reduce error on identifying fabric defects requires automotive and accurate inspection process(Vikrant and Gaurav2013). Considering this lacking, this research implements a textile defect detector. A multi-layer neural network is used to detect defects for the specific problems. The input fabric images for the neural network is captured byadigitalcamera. The color images are first converted into binary images by restoration process and local threshold techniques, then three different features are determined for the actual input to the neural network, which are the area of the defects, number of the objects in a image and finally the shape factor. The developed system is able to identify two very commonly defects such as holes and scratches and other types of minor defects. The developed system is very suitable for least developed countries, identifies the fabric defects within economical cost and produces less error prone inspection system in realtime.A feed forward neural network was considered for training with error back propagation algorithm. The neural network was trained properly on training datasets with predefined goal. The unseen test dataset was presented to neural network after training for classification purpose (Liu Zhang and Metaxas 2016).In the manual fault detection system with highly trained inspectors, very less percentageofthedefects on fabrics isbeing detected in thetextileindustries. But a real time automatic system can increase this percentage in a maximum number. This research implements a textile defect detector which uses computer visionmethodologywiththehelpofneuralnetworkstoidentifyt heclassification of textile defects (Lois $M$ Hoffer and Giuseppe Longobardi1996).

\section{IMAGE PROCESSING}

An image may be defined as a two-dimensional function, $f(x, y)$, where $x$ and $y$ are spatial (plane) coordinates, and the amplitude off at any pair of coordinates $(\mathrm{x}, \mathrm{y})$ is called the intensity or gray level of the image at that point. When $\mathrm{x}, \mathrm{y}$, and the amplitude values of $\mathrm{f}$ are all finite, discrete quantities, we call the image a digital image. The field of digital image processing refers to processing digital images by means of a digital computer. Note that a digital image is composed of a finite number of elements, each of which has a particular location and value.These elements are referred to as picture elements, image elements, pels, or pixels. Pixel is the term most widely used to denote the elements of a digital image ( Lal, Ajay, and Ankit2013)

\section{ARTIFICIAL NEURAL NETWORK}

The Artificial Neural Networks (ANN) is inspired by the way biological nervous system works, such as brain processes an information. ANN mimics models of biological system, which uses numeric and associative processing. In two aspects, it resembles the human brain. It acquired knowledge from its environment through a learning process. Synaptic weights, used to store the acquired knowledge, which is interneuron connection strength. Here Convolutional neural network is used (Rebhi ,Issam and Farhat2015).

\section{A. CONVOLUTIONAL NEURAL NETWORK}

In deep learning, a ConvolutionalNeural Network (CNN) is a class of deep neural networks, most commonly applied to analyzing visual imagery. They are also known as shift invariant or space invariant artificial neural networks (SIANN), based on their shared-weights architecture and translation invariance characteristics. They have applications in image and video recognition, recommender systems, image classification, medical image analysis, natural language processing, brain-computer interfaces, and financial time series (Huanhuan, JinxiuJunfengand Pengfei2019).The aim of the present work is to identify different types of defects in woven fabric and analyzing by using Convolutional Neural Network.

\section{METHODOLOGY}

In this research, Digital Image Processing application is applied for defected fabric. The research is based on the quality images of the defected fabrics. The various defective fabric images are captured by the digital camera and categorized in to different defect groups to create a data set. The neural network has been trained with the raw images. Then the trained network was used to detect or classify the defects in the testing samples. The accuracy of the classification is computed from the Test results. The training and testing was carried through the online portal Google's teachable machine (Figure 1). The samples are uploaded into the cloud from the dataset and named as Snarls, Stain, Wrong drawing, Selvedge tails and Holes respectively. Thenetwork parameters like epoch and goal are set to train the Network. Then the Training has been done using the uploaded dataset to create network. Once the Training has been done successfully, each sample was tested one by one to get the results for classification. Data flow diagram of the process and the flow process in training is given in Figure 2 and 3.

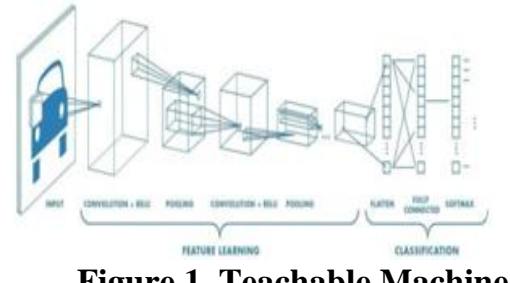

Figure.1. Teachable Machine 

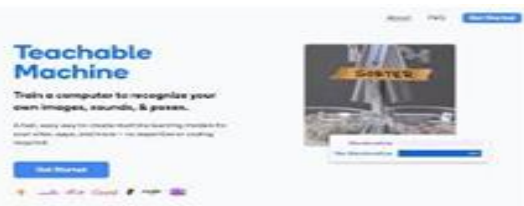

Figure.2. Data flow diagram of the process

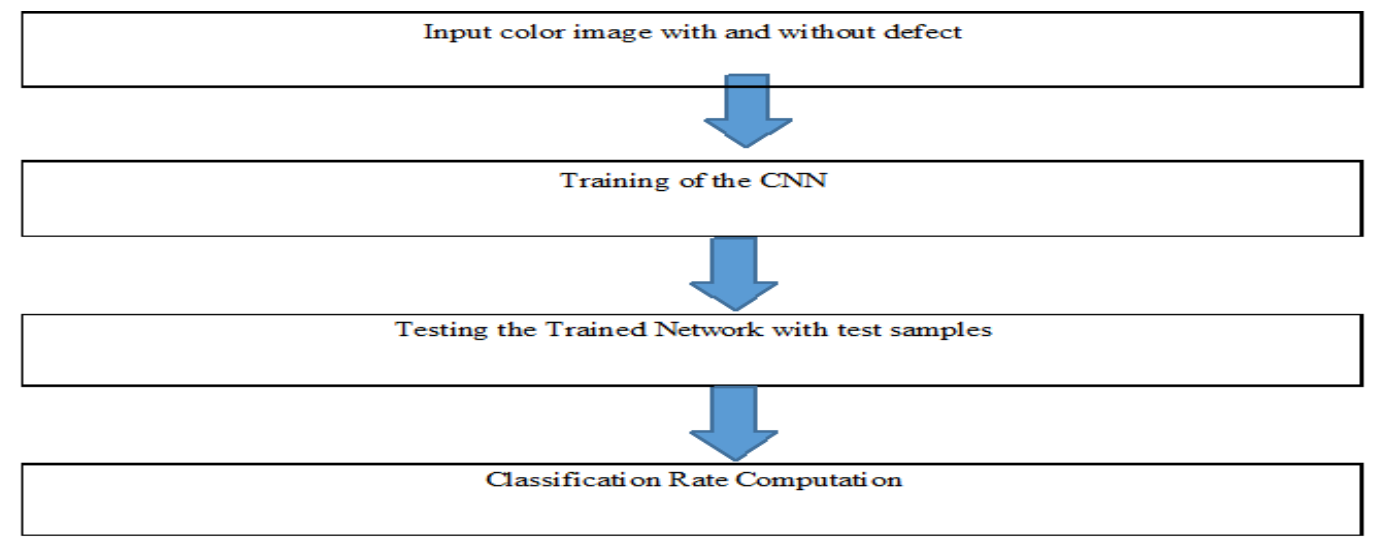

Figure.3.Flow process in Training

\section{DATASET}

Thesampleswithdefects were collected fromSkyCotexIndia Pvt.

Ltd,Tirupur.

Thecoloredimagesofthefabricweregatheredfromthehighquali ty

imagesensingdevice.Theimagesweretransferredfromimagese nsingdeviceto the computer system. 300 images were collected through the image acquisition process. From the Sample Images, 150 images are used for training the Network and remaining 150 images are used for testing. Figure 4 (a),(b),(c),(d) represents the image samples for the defects such as Snarl, Stain, Wrong drawing, Selvedge tails and Holes on the fabric respectively.

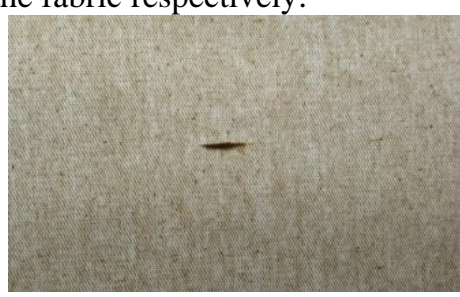

(a)

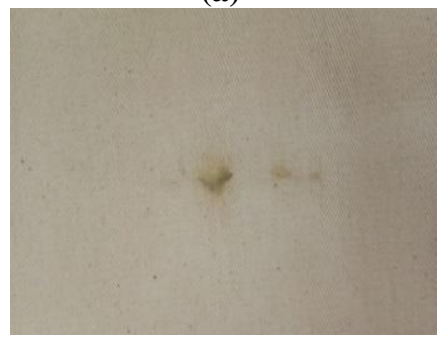

(b)

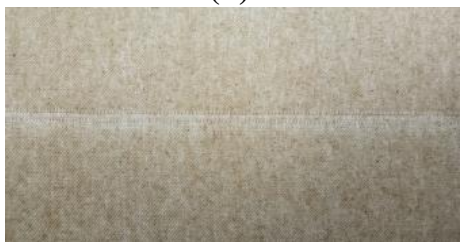

(c)

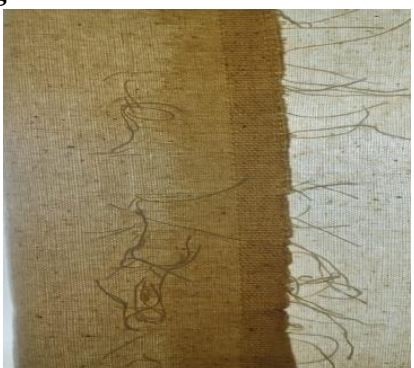

(d)

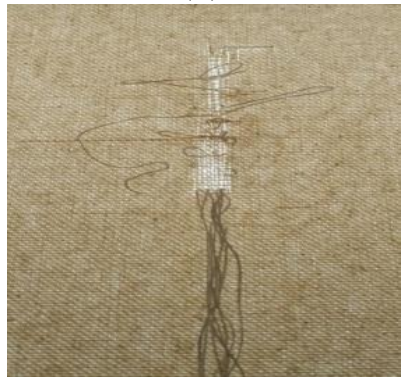

(e)

Figure.4.(a), (b), (c), (d) and (e) represent the defects such as Snarl, Stain, Wrong drawing, Selvedge tails and Holes.

\section{TRAINING OF THE CONVOLUTIONAL NEURALNETWORK}

TheNeuralNetworkwastrainedusing150images and remaining 150 images were used for testing. The Figure 5 shows the architecture of the Convolutional Neural Network. The Convolutional Neural network consists of Input layer and hidden layers and output layer. The number of neurons in the Input layers depends on the size of the Input image. Fortrainingtheneuralnetwork,the epochs and learning rate, goal were set and trail has been conducted by varying the epochs to obtain better classification rate.

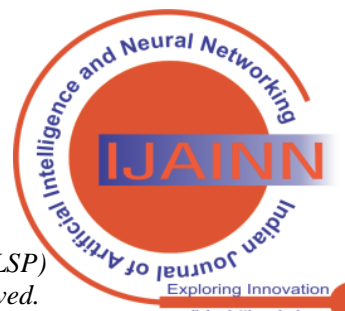




\section{Deep Learning Convolutional Neural Network for Defect Identification and Classification in Woven Fabric}

The number of epochs is a hyperparameter that defines the numberof times that the learning algorithm will work through the entire training dataset. One epoch means that each sample in the training

dataset has had an opportunity to update the internal model parameters.If epoch's value is set to 50, for instance, it means that the model which isunder training will work through the entire training dataset 50times (Subrata Das and Amitabh Wahi2019). Generally the larger the number, the better is the model will learn to predict the data and greater is the accuracy. Generally, one wants to tweak (usually increase) this number until good predictive results are achieved with the model. The value of epoch used was 50 for getting a good result and the learning rate was fixed as0.001.The Figure 6 shows the training neural network through the Teachable machine portal.
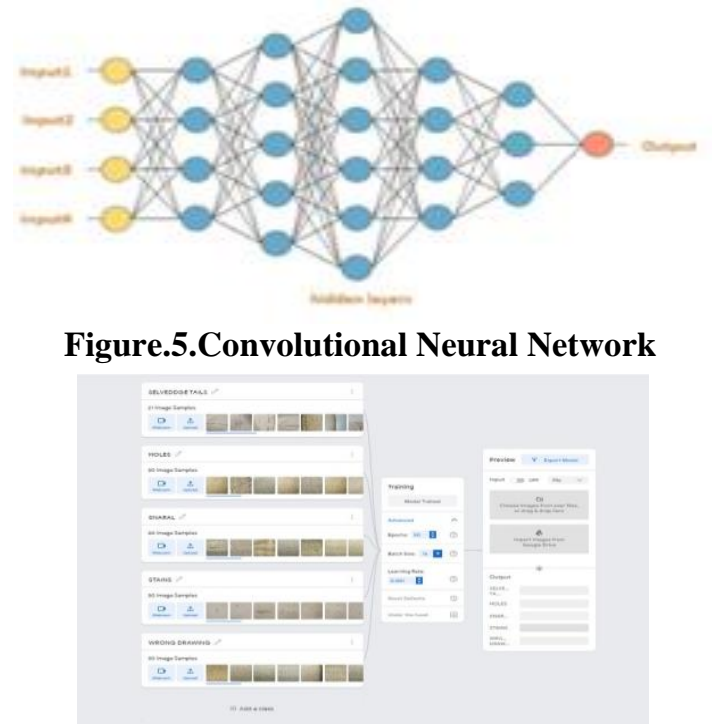

Figure.6.Training Neural Network

CNN Parameters for Training the Network:

Epochs- 50

Batch Size- 16

Learning Rate- 0.001

Time taken for Training CNN was $36 \mathrm{sec}$

\section{TESTING NEURAL NETWORK}

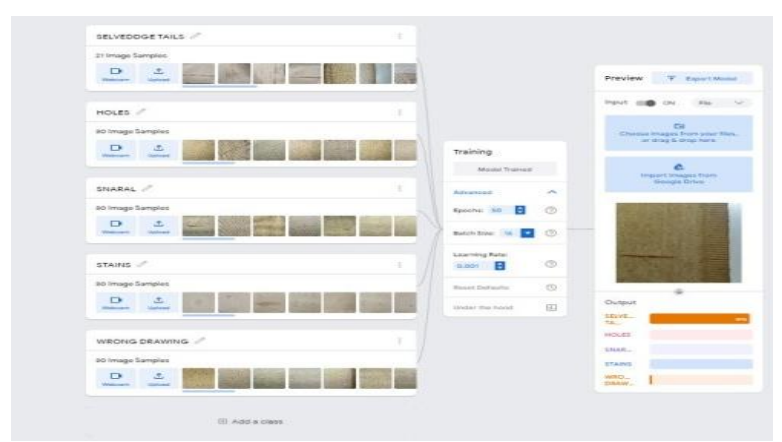

Figure.7. Testing Neural Network

After training the neural network using images collected and remaining images are used for testing. The images are uploaded in the testing section orderly and tested to get accuracy percentage which accordingly gives the efficiency of the trained neural network .These images usedtocalculatetheefficiencypercentage of the training machine and the neural network. The Figure 7 shows the testing the trained neural network through the Teachable machine portal.

\section{EXPERIMENTAL RESULT}

\section{CONFUSION MATRIX}

A confusion matrix shown in figure 8 summarizes how accurate your model's predictions are. This matrix to figure out which classes the model gets confused about. The y axis (Class) represents the class of your samples. The $\mathrm{x}$ axis (Prediction) represents the class that the model, after learning, guesses those samples belong to. So, if a sample's Class is "Muffin" but its Prediction is "Cupcake", that means that after learning from your data, the model misclassified that Muffin sample as a Cupcake. This usually means that those two classes share characteristics that the model picks up on, and that particular "Muffin" sample was more similar to the "Cupcake" samples.

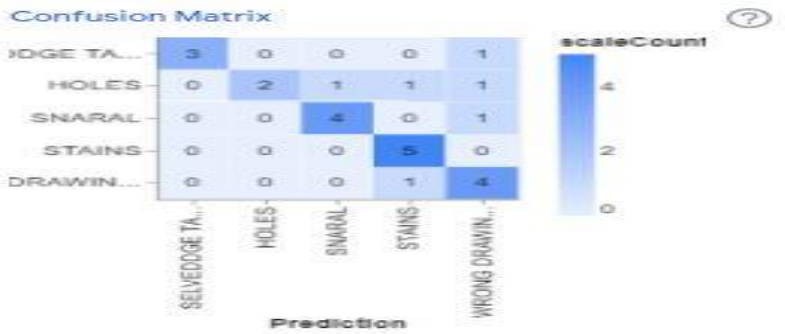

Figure.8.Confusion matrix

TABLE I- Experimental Resultsof Defects afterTesting

\begin{tabular}{|c|c|}
\hline Defect Names & Classification Percentage $\%$ \\
\hline HOLES & 78.1 \\
\hline STAINS & 81.6 \\
\hline SELVEDGE TAILS & 84.7 \\
\hline WRONG DRAWING & 84.8 \\
\hline SNARL & 74.6 \\
\hline
\end{tabular}

\section{A. Accuracy per epoch}

Accuracy is the percentage of classifications that a model gets right during training. If the model's prediction is perfect, the accuracy is one; otherwise, the accuracy is lower than one. The accuracy per epoch is shown in the figure 9 .

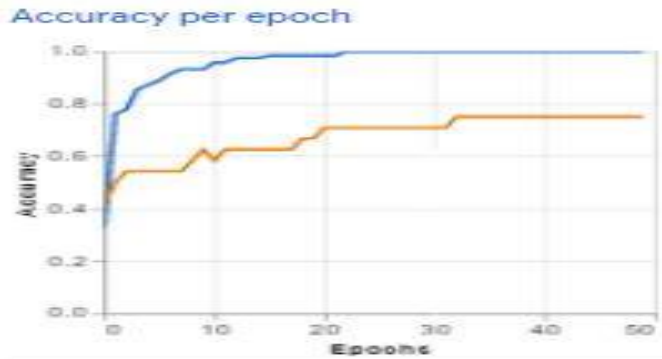

Figure.9.Accuracy per epoch

\section{B. Loss per epoch}

Lossisameasureforevaluatinghowwellamodelhaslearnedtop redicttheright classifications for a given set of samples. If

the model's predictions are perfect, the loss is zero; otherwise, the loss is greater than

zero.

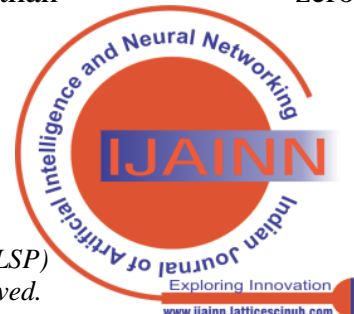


Both models have the same accuracy, but model without defect has a lower loss value as shown in Figure 10.

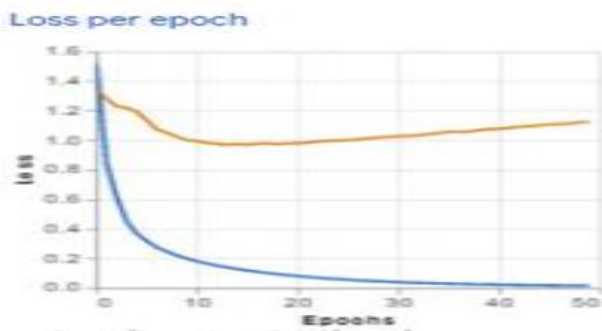

Figure 10. Loss per epoch

\section{A. Efficiency}

The images were tested in the trained neural network. The efficiency for five different defects in woven fabric is tested and calculated. 20 random samples were taken from each defect data set and efficiency is derived and average is taken. The efficiency percentage of holes, stains, selvedge tails, wrong drawing and snarl is $78.1 \%, 81.6 \%, 84.7 \%, 84.8 \%$ and $74.6 \%$. The experimental results after training and testing are given in the Table I.

\section{CONCLUSION}

Images were collected and the neural network was trained and tested using teachable machine and the efficiency was calculated for each defect class and finally averaged out.The automatic defect identification in the woven fabric based on Convolution Neural Network (CNN) in Deep Learning was achieved and the classification accuracy in percentage of holes, stains, selvedge tails, wrong drawing and snarl are $78.1 \%, 81.6 \%, 84.7 \%, 84.8 \%$ and $74.6 \%$ respectively.The productivity and quality of the apparels will be increased to a greater extent if this kind of automatic defect detection and identification system is applied in inspection systems of apparel industry.

\section{REFERENCE}

1. Bay, H., T. Tuytelaars, and E. Van gool. Surf speeded up robust features. In computervision-eccvSpringer,2006, pp.404-417. [CrossRef]

2. Borghese, N.A., andS. Fomasi. Automatic defect classification on a production line. Intelligent industrial systems ,2015,pp. 443-46. [CrossRef]

3. Chan, C., and G. K. H. Pang.Fabric defect detection byFourieranalysis.IEEE Trans. on Ind,2000, Appl4 ,pp.267-76.

4. Everingham, M.,S.A.Eslami,L.Van gool, C.K.Williams, J. Winn, and A. Zisserman.Thepascalvisualobjectclasseschallenge:Aretrospective. International journal of computer vision,2015, pp.137-39.

5. Goyal, A. Automation in fabric inspection, in automation in garment manufacturing.Woodhead publishing,2018 ,pp.140-43. [CrossRef]

6. Habib, T., C. Faisal, R.Rokonuzzaman, and M. Ahmed. Automated fabric defect inspection: a survey of classifiers. International journal in foundations of computer science \& technology (IJFCST), 2014,pp.17-25. [CrossRef]

7. Hillel , R. Lerner, D. Levi, and G. Raz. Recentprogressinroad and lane detection: a survey. Machine vision and applications, 2014,pp.10-21.

8. Huanhuan, Z., M. Jinxiu, J. Junfeng, and L. Pengfei. 2019. Fabric defect detection using LO gradient minimization and fuzzy C-means. Applied Sciences ,pp.3506-10. Available : doi:10.3390/app9173506. [CrossRef]

9. İbrahim, Ç. H., D. L. Canan, and T. Mehmet. 2016. Application of neural networks (NNs) for fabric defect classification ,pp.221-49. Available : doi:10.5772/63427. [CrossRef]

10. Kang T.J. et al.Automatic Structure Analysis and Objective Evaluation of Woven Fabric Using Image Analysis. Textile Res. J,2001,pp.261-70. [CrossRef]

11. Kumar, A. 2003. Neural network based detection of local textile defects. Pattern Recognition ,pp.1645-59. Available: doi:10.1016/S0031- 203(03)00005-0. [CrossRef]

12. Lal, R. J., B. Ajay, and C. Ankit. 2013. Real time fabric defect detection system on an embedded DSP platform,pp.5280-84 Available :doi:10.1016/j.ijleo.03.038. [CrossRef]

13. Li, Y., W. Zhao, and J.Pan .Deformable patterned fabric defect detection with fisher criterion-based deep learning. IEEE Transactions on Automation science and Engineering , 2017,pp.1256-64. [CrossRef]

14. Liu, J.,S. Zhang, S. Wang, and D.N. Metaxas Multispectral deep neural networks for pedestrian detection , 2016, pp.155-59. [CrossRef]

15. Long, J., E. Shelhamer, and J. Darrell. Fully convolutional networks for semantic segmentation.In proceedings of the IEEE conference on computer vision and pattern recognition,2015 ,pp. 3431-40. [CrossRef]

16. Lois, M. H., F. Franco ,T. Bruno, and L. Giuseppe. Neural networks for the optical recognition of defects in cloth, 1996,pp.3183-90 [CrossRef]

17. Rebhi, A., I. Benmhammed, S. Abid, and F. Fnaiech. Fabric defect detectionusinglocalhomogeneityanalysisandneuralnetwork.Journalof photonics, 2015,pp.10-13. [CrossRef]

18. Rebhi, A., B. Issam, A. Sabeur, and F. Farhat. 2015. Fabric defect detection using local homogeneity analysis and neural network. Journal of Photonics,pp.1-9. Available :doi:10.1155/2015/376163. [CrossRef]

19. Sari-Sarraf, H.,and J. S. Goddard. Vision system for on-loom fabric inspection.IEEE transactions on industry applications , 1999,pp.1252 59. [CrossRef]

20. Subrata, D., A. Wahi, S. Keerthika, N. Thulasiram, and S. Sundaramurthy. Automated defect detection of woven fabric using artificial neural network. Man Made Textiles in India .2019,pp.11315.

21. Vikrant, T., and S. Gaurav. Automatic fabric detection using morphological operations on bit plane. International Journals of Engineering Research \&Technology , 2013,pp.856-61.

22. Wang, T., Y. Chen, M. Qiao, and H. Snoussi. 2018. A fast and robust convolutional neural network-based defect detection model in product quality control. The International Journal Advanced Manufacturing Technology ,pp.3465-71. Available:doi:10.1007/s00170-017-0882-0. [CrossRef]

23. Wiltschi, K., A. Pinz, and T. Lindeberg. 2000. Automatic assessment scheme for steel quality inspection. Machine Vision and Applications ,pp.113-28.Available : doi:10.1007/s001380050130. [CrossRef]

24. Zhang, X. F., and R. R. Breese. Fabric defect detection and classification using image analysis. Textile Res. J65, 1995,pp.1-9. [CrossRef]

\section{AUTHORS PROFILE}

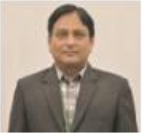

Dr. Subrata Das, PhD degree from I.I.T., Delhi, India. He is a Professor of Fashion Technology at B.I.T., Sathyamangalam, TN, India. He is a Technical Expert, GOTS and OCS audit and Inspection, under NABCB, Govt of India.

A. Ms.Aiswarya Mohan, student of Bannari Amman Institute of Technology pursuing Fashion Technology, having interest in Artificial intelligence.

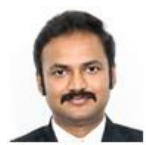

Dr. S Sundara Murthy is an Associate Professor in the department of Information Technology at the Bannari Amman Institute of Technology. The principle areas of research in which the author has worked are Artificial Intelligence, Convolution Networks, Neural Networks , Computer Vision, Digital Image Processing.

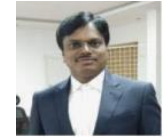

Mr.Suresh Jayaram is one of the Directors of Skycotex India pvt ltd. leading manufacturers of Yarn, Fabrics and Institutional textile products. As a pioneer himself the author has expertise in clothing sector by adapting to the emerging trends and helping the student scholars in various aspects of their research works. 